\title{
Two cases of osteomyelitis due to group $G$ streptococcus
}

NIRMAL JOSHI, MD, JOHN N GOLDMAN, MD

\begin{abstract}
Two cases of group G streptococcal osteomyelitis in adults are reported, one of the patients being an otherwise healthy individual. Both cases were successfully treated with parenteral aqueous penicillin G. Only one case of group G streptococcal osteomyelitis
\end{abstract}

has been described previously in the literature; this was in an intravenous drug abuser. Can $J$ Infect Dis 1990;1(2):61-63

Key Words: Group G streptococcus, Osteomyelitis
Streptococci belonging to group g have been Sinvolved in a variety of infections in adults, ranging from bacteremia and endocarditis (1) to surgical wound infections (2), septic arthritis (3) and endophthalmitis (4). To the authors' knowledge there has been only one case reported in the literature of osteomyelitis due to group G streptococcus in adults in the absence of septic arthritis, this being in an intravenous drug abuser (5). Two cases of group G streptococcal osteomyelitis are reported, one of them in an individual with no underlying medical disorders.

Division of Infectious Diseases and Epidemiology. Department of Medicine, The Milton S Hershey Medical Center, University Hospital, The Pennsylvania State University, Hershey, Pennsylvania, USA

Correspondence and reprints: Dr Nirmal Joshi, Division of Infectious Diseases and Epidemiology, Department of Medicine, The Milton S Hershey Medical Center, University Hospital, The Pennsylvania State University, Hershey, PA 17033, USA. Telephone (717) 531-8881

Received for publication February 6, 1990. Accepted May 7, 1990

\section{CASE PRESENTATIONS}

Case 1: This 58-year-old man had sustained multiple injuries in a car accident two years previously, including a left tibial fracture for which open reduction and internal fixation was done. He was subsequently ambulatory and doing well until two weeks prior to admission when he developed redness and swelling on the medial aspect of the left ankle. Local $x$-rays done at this time were normal except for the presence of a K-wire in the distal left tibia (used for internal fixation). He was treated with oral cephalexin for presumed cellulitis, but the swelling worsened and he was admitted to the hospital. He had no history of drug abuse, homosexuality or recent trauma.

On physical examination he had a temperature of $36.5^{\circ} \mathrm{C}$, with swelling, redness and tenderness on the medial aspect of the left ankle in the region of the distal tibia.

Movement at the ankle joint was full and painless. Pertinent laboratory data included a peripheral leukocyte count of $10,500 / \mathrm{mm}^{3}$ with a normal differential count and a Westergren 
sedimentation rate of $67 \mathrm{~mm} / \mathrm{h}$. Drainage was performed and revealed subcutaneous pus and an abscess cavity in the distal tibia. The K-wire was not close to the operative field, being lateral to the abscess cavity. Its removal would have required considerably more tissue dissection, which was not considered necessary. Gram stain of the pus from the tibial abscess revealed Gram-positive cocci in chains, and culture revealed a pure growth of group G streptococci. Anaerobic cultures were negative. He was treated with parenteral aqueous penicillin $\mathrm{G}$ to which the organism was sensitive (minimal inhibitory concentration [MIC] less than $0.03 \mu \mathrm{g} / \mathrm{mL}$ ). This drug was administered intravenously at a dose of two million units every $4 \mathrm{~h}$ for four weeks, followed by oral phenoxymethylpenicillin at a dose of $500 \mathrm{mg}$ qid with probenecid $500 \mathrm{mg} 30 \mathrm{mins}$ before each penicillin dose for another 12 weeks. At 12 weeks of follow-up, the patient was clinically well with no fever or recurrence of local swelling or pain. Plain $\mathrm{x}$-rays showed good healing with no evidence of recurring osteomyelitis.

Case 2: This 70-year-old man had a history of oral squamous cell cancer treated with resection and local radiation four years previously. He presented with worsening low back pain beginning two weeks after initiation of radiation therapy for recurrent tumour two months before admission. He had also noted fever of up to $38.9^{\circ} \mathrm{C}\left(102^{\circ} \mathrm{F}\right)$ for several weeks. He denied any bladder or bowel complaints.

Physical examination revealed a temperature of $38.8^{\circ} \mathrm{C}$ and mild right paraspinal tenderness in the lumbar area with no local deformity and no focal neurological findings. Examination of the oral cavity did not reveal an obvious focus of infection or inflammation.

Pertinent laboratory data included a peripheral leukocyte count of $7900 / \mathrm{mm}^{3}$ with $54 \%$ neutrophils and 25\% bands, and a Westergren sedimentation rate of $120 \mathrm{~mm} / \mathrm{h}$.

Radiograph of the lumbar spine revealed total destruction of the disc space between L2 and L3 with bony destruction of the inferior part of L2. Radionuclide bone scan using technetium-99 methylene diphosphonate showed markedly increased activity in the same area. A computed tomography scan-guided bone biopsy and culture of tissue was obtained. Pathologic examination of the bone revealed extensive acute and chronic inflammation and granulation tissue. No tumour or granuloma were seen. Gram stain of the specimen did not reveal any organisms, but culture revealed a pure growth of group G streptococci sensitive to penicillin (MIC less than $0.03 \mu \mathrm{g} / \mathrm{mL}$ ). He was treated with parenteral aqueous penicillin $\mathrm{G}$ at a dose of two million units every $6 \mathrm{~h}$ for eight weeks postoperatively and also placed in a spica body jacket. He did well, with improvement in radiologic changes and no evidence of recurrent osteomyelitis at two-and-one-half years of followup.

Microbiological methods: Group G streptococci in both cases were identified in the laboratory by beta-hemolysis on sheep blood agar, negative catalase test, negative bile esculin test and resistance to bacitracin disc. Specific Lancefield grouping was performed using the rapid latex agglutination method (Wellcome Reagents). MIC testing was done by broth microdilution technique (PASCO Laboratories, Wheat Ridge, Colorado).

\section{DISCUSSION}

Streptococci belonging to group $\mathrm{G}$ were first identified by Lancefield and Hare in 1935 (6) and at that time were not considered pathogenic. Since then, however, they have been associated with a number of diseases, both in animals and humans. Infections caused by these organisms in animals, unlike those caused by group $\mathrm{C}$ streptococci, are uncommon. They include bovine mastitis (7), necrotic dermatitis in mice (8), pneumonia in sheep (9) and cervical adenopathy and foot abscesses in cats (10).

Asymptomatic humans may harbour these organisms in the skin, pharynx and gastrointestinal tract. They have also been recovered from the vaginas of asymptomatic puerperal females (12). They are known to cause a number of infectious diseases in humans (1-5). Most of these patients have had some predisposing factor: alcoholism, drug addiction, diabetes mellitus or carcinoma. The incidence of infections caused by these organisms at the authors' hospital (a 350-bed university teaching hospital) remains low. Over a two year period, they were isolated from the blood of three patients, the ascitic fluid of one and the synovial fluid culture of one.

Group G streptococci are identified by means of beta-hemolysis on blood agar, inability to grow in the presence of bile and agglutination with group specific antisera following antigen extraction (11). Most infections respond to therapy with penicillin, although addition of gentamicin in patients with endocarditis has been suggested to improve outcome (1).

Except for one case report of group G streptococcal osteomyelitis in an intravenous drug abuser (5), these two cases are the first cases of osteomyelitis described due to this organism. Case 1 is especially noteworthy for the occurrence of osteomyelitis in a previously healthy individual, the only apparent predisposing factor being old local trauma. It is likely that the organism gained 
entry at the time of initial trauma or subsequent surgery. The later occurrence of osteomyelitis may have been due in part to local compromise from the initial fracture or the ongoing presence of hardware. A predilection of patients with cancer to develop group $\mathrm{G}$ streptococcal disease has been described earlier (12), and case 2 would support this association. A recent case report describes an

\section{REFERENCES}

1. Smyth EG, Pallett AP, Davidson RN. Group G streptococcal endocarditis: Two case reports, a review of the literature and recommendations for treatment. J Infect 1988;1B:169-76.

2. Brahmadathan KN, Koshi G. Importance of group G streptococci in human pyogenic infections. J Trop Med Hyg 1989;92:35-8.

3. Fujita NK, Lau K, Bayer AS. Septic arthritis due to group G streptococcus. JAMA 1982;247:812-3.

4. Berkey PB, Rolston KV. Group G streptococci as a cause of bacterial endophthalmitis. Case report. Arch Ophthalmol 1988;71:198-202.

5. Chandrasekar PH, Narula AP. Bone and joint infections in intravenous drug abusers. Rev Infect Dis 1986;8:904-11.

6. Lancefield RC, Hare R. The serological differentiation of pathogenic and nonpathogenic streptococci from parturient women. J Exp Med 1935;61:335-49.

7. Hamilton CA, Stark DM. Occurrence and characterization of Lancefield group G streptococci in apparently otherwise healthy young man with group $\mathrm{G}$ streptococcal bacteremia (13). It is possible that in case 2 , the source of the organism may have been the oral cavity, and spread resulted from a breach in the mucosa secondary to radiation therapy.

These cases add to the known spectrum of disease caused by group $\mathrm{G}$ streptococci.

bovine mastitis. Am J Vet Res 1970;31:397-8.

8. Stewart DD, Buck GE, McConnell EE, et al. An epizootic of necrotic dermatitis in laboratory mice caused by Lancefield group G streptococci. Lab Anim Sci 1975;25:296-302.

9. Krantz GE, Dunne HW. An attempt to classify streptococcal isolates from domestic animals. Am J Vet Res 1965;26:951-9.

10. Goldman, PM, Moore TD. Spontaneous Lancefield group $\mathrm{G}$ streptococcal infection in a random source cat colony. Lab Anim Sci 1973;23:565-6.

11. Blair DC, Martin DB. Beta hemolytic streptococcal endocarditis: Predominance of non-group A organisms. Am J Med Sci 1978;276:269-75.

12. Auckenthaler R, Hermans PE, Washington JA. Group G streptococcal bacteremia: Clinical study and review of the literature. Rev Infect Dis 1983;5:196-204.

13. Teel LD, Shemonsky NK, Aronson N. Group G streptococcal bacteremia in a healthy young man. South Med J 1989;82:633-4. 


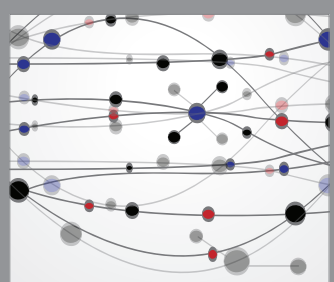

The Scientific World Journal
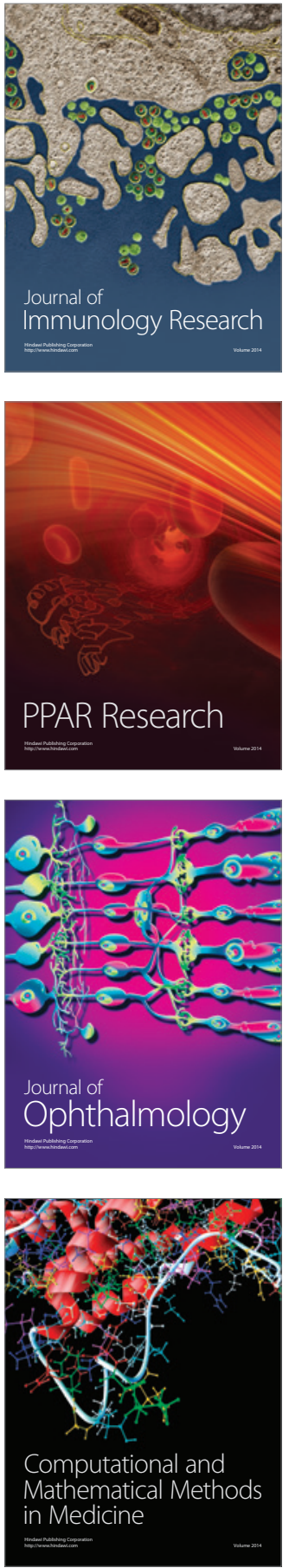

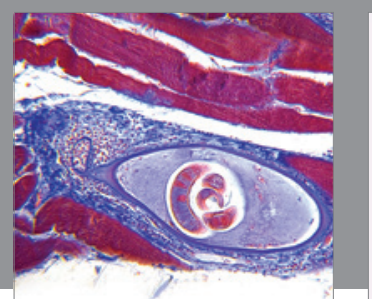

Gastroenterology Research and Practice

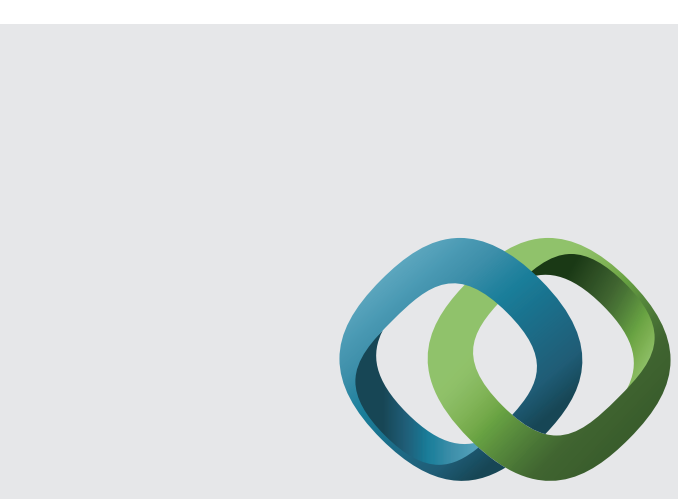

\section{Hindawi}

Submit your manuscripts at

http://www.hindawi.com
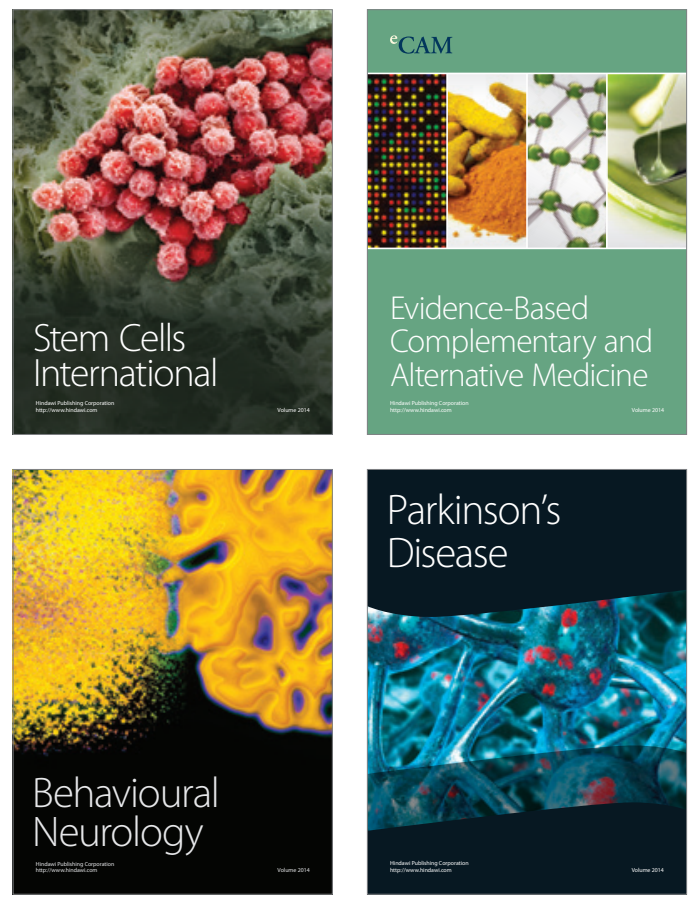
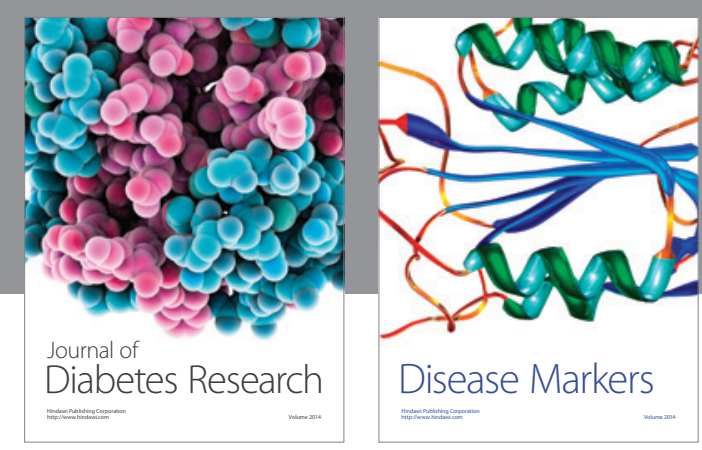

Disease Markers
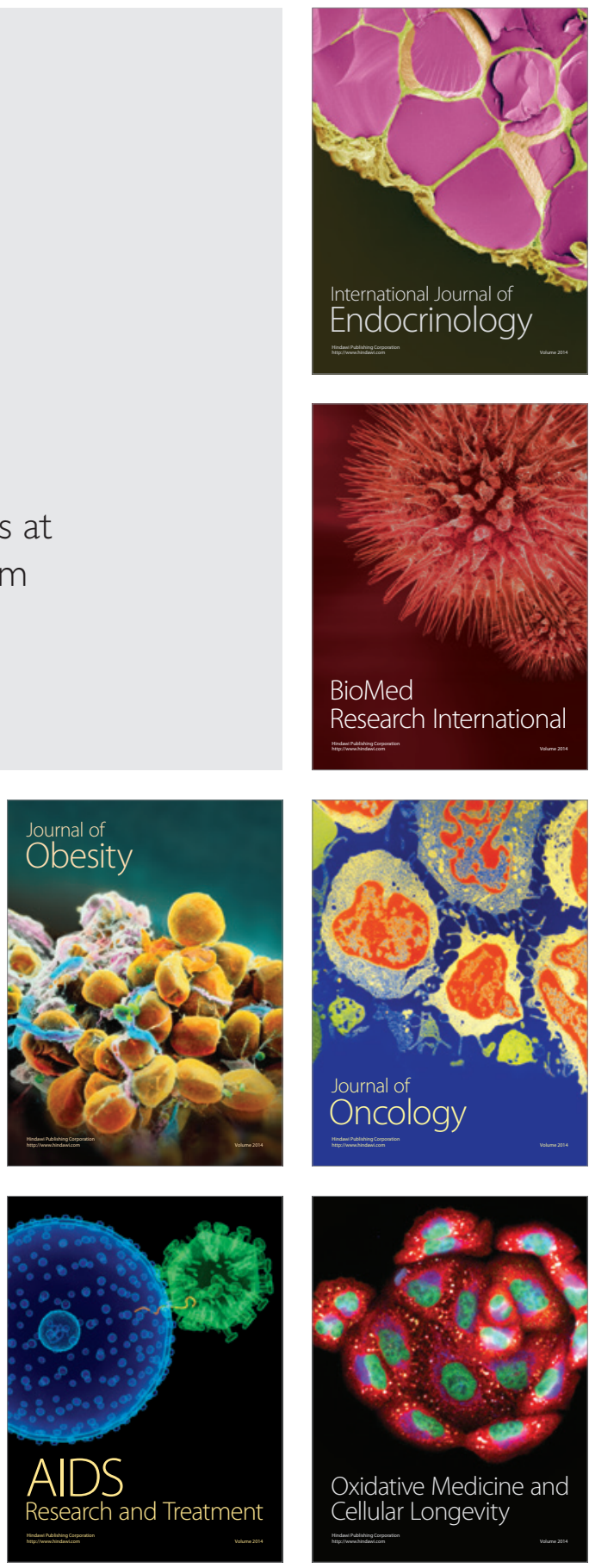